\title{
Overlapped and Sequential Metasurface Modulations for Bi-Chromatic Beams Generation
}

\author{
Marco Faenzi ${ }^{1}$, David González-Ovejero², Stefano Maci ${ }^{1}$ \\ (1) Department of Information Engineering and Mathematics, University of Siena, 53100 Siena, Italy \\ (2) Univ Rennes, CNRS, IETR (Institut d'Électronique et des Technologies du numéRique) - UMR 6164, F-35000, Rennes, France \\ (david.gonzalez-ovejero@univ-rennes1.fr)
}

\begin{abstract}
This paper describes the generation of directive beams at two different frequencies with the same circular metasurface (MTS) antenna based on a surface wave (SW) excitation. Two methods are presented to achieve the desired bi-chromatic beam operation. The first one consists in mathematically superimposing two MTS modulations, each one matched to the SW wavelength at a different frequency. In the second method, the period of the MTS modulation matches the SW wavelengths at two different frequencies in two different regions of the antenna, i.e., close to the center for the high frequency band and close to the periphery for the low frequency band. The first method allows one to have different feed points for each beam in a self-diplexed structure, although it is also possible to use a single feed configuration and a diplexer. The second approach only holds for a single physical feed-point. Numerical and experimental results are shown.
\end{abstract}

Index Terms—-Dual-band, leaky wave (LW), metasurface, surface wave (SW).

Metamaterial research has gained renewed interest due to the advent of bi-dimensional metamaterials, most commonly known as metasurfaces (MTSs). The initial focus on non-modulated (uniform) MTSs ([1] and references therein) has been reoriented to modulated MTS both for optics and microwaves. Modulated MTSs have been recently used to tailor surface-wave (SW) and plasmonic wave-fronts [2]-[3], to control field transmission [4], realizing anomalous reflections [5], and to control surface-to-leaky wave transformation [6]-[8]. This paper deals with the latter case, which has a significant applicative impact on low-profile microwave antennas. These antennas consist of a SW launcher, which couples the input power to a transverse magnetic (TM) SW, and a planar aperture made of sub-wavelength elements, which can be homogenized as an equivalent inductive impedance surface. The SW power is gradually radiated by modulating the surface inductance. Indeed, the SW electric field multiplied by the periodically modulated impedance, provides a discrete spectrum of Floquet-wave sheets, where the -1 indexed term enters the visible spectral region. Such modulation can be implemented by modifying the height and orientation of metallic pillars [9] or by changing the size and orientation of sub-wavelength patches printed on a grounded dielectric slab. Modulated MTSs based on subwavelength patches have been applied to obtain high aperture efficiencies [10], high polarization purity [8], beam shaping [11], and dual polarization [11]. The gain-bandwidth product (GBWP) of MTS antennas is impaired by the SW dispersivity, and this product is proportional to the SW group-velocity $\left(v_{\mathrm{g}}\right)$ and the electrical size of the aperture [12]. Nonetheless, MTS antennas are not necessarily narrow-band [12]. Indeed, it has been shown in [13]-[14] that their bandwidth may be significantly increased at the expense of reducing the aperture efficiency, while maintaining the GBWP limited by the average group-velocity over the band. However, in some specific applications (like satellite communications at Ka-band), rather than a large bandwidth, the same aperture must provide two separate sub-bands that sometimes are considerably separated from each other. Therefore, the antenna gain could be significantly increased with respect to wideband designs when the aperture operates only at two different frequencies. A first attempt to obtain dual-band operation was reported in [15] for conical beams, which were obtained using a backward-mode and a 
forward-mode at the low and high frequency bands, respectively. The superposition of impedance modulations was proposed in [16] for multi-beam operation and applied in [17] to design a dual-band MTS antenna; a similar approach was used in [18] to generate two beams at 17 and 20GHz. Multi-frequency synthesis has recently been proposed in [19], where a direct inversion of the electric field integral equation in [20], [21] was used to generate beams at 20 and $24 \mathrm{GHz}$. Finally, the superposition of holograms was used in [22] to record onto a single metasurface hologram two independent holographic images at high and low spatial frequencies. These two holographic images can be separated from the reconstructed overlapped images by using two digital Gaussian filters.

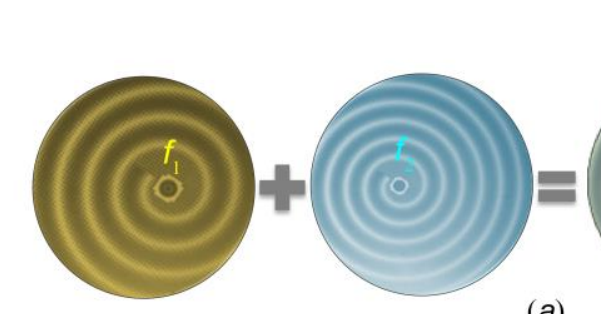

(a)
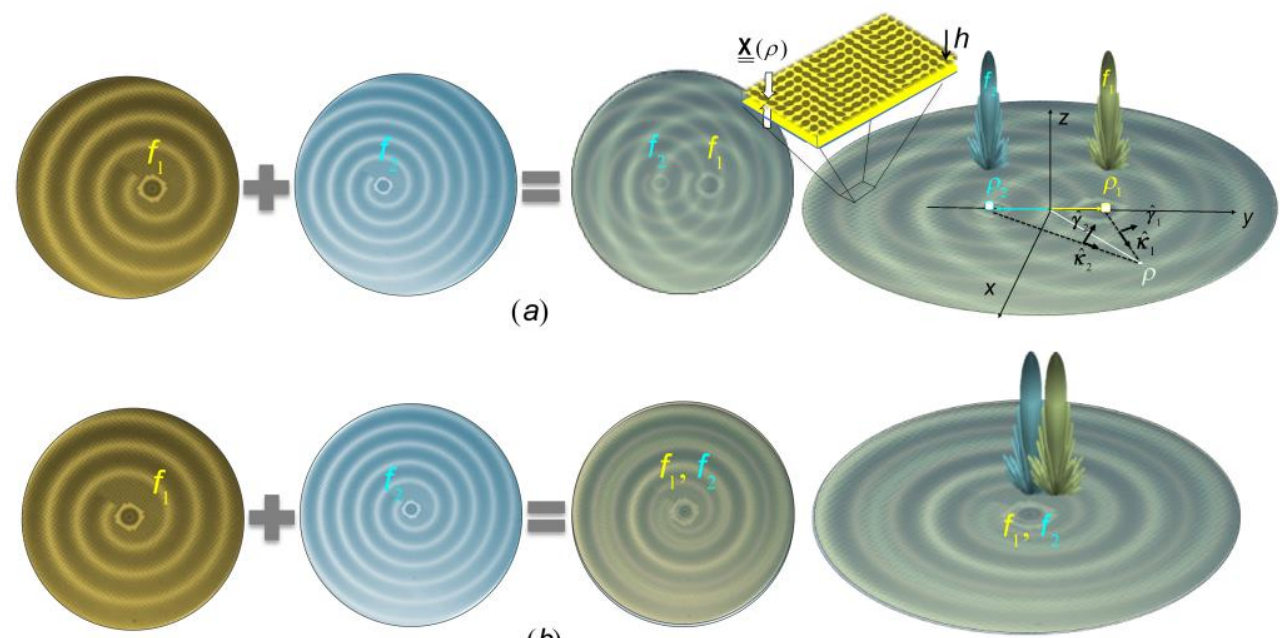

(b)
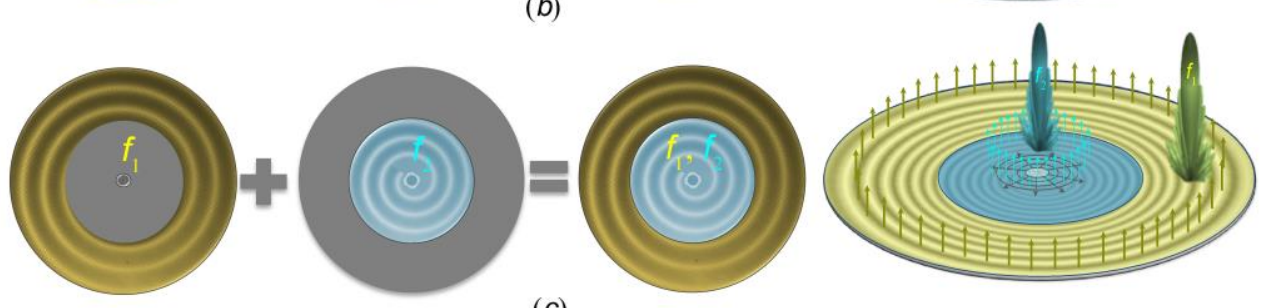

(c)

Fig. 1. Basic mechanisms for bi-chromatic beam generation. (a) Overlapping of aperture modulations with two different feeds; (b) overlapping of aperture modulations with one feed only, as a special case of (a); (c) sequential modulation with two regions. Top right: reference coordinate system used to define the reactance in case (a). In the inset: printed subwavelength elements on a grounded substrate used to implement the resulting modulated impedance. Bottom right: annular regions pertinent to the generation of the two individual beams. The impedance changes with continuity from the center to the periphery of the antenna.

In this paper, we present and compare two different strategies, schematically shown in Fig. 1, to obtain bichromatic beams pointing in the broadside direction. In Fig. 1a, the first modulation presents a period that provides beam \#1 at frequency $f_{1}$, while the period of the second modulation provides beam $\# 2$ at frequency $f_{2}$. The resulting inductive modulated surface is then synthesized by means of sub-wavelength patches printed on a grounded dielectric slab. In Fig 1a, a different position has been chosen for the origin of each modulation to better decouple the mutual interaction between the excitation at one frequency and the modulation pertinent to the other frequency. The two separated feedpoints enable self-diplexing of the signals received at $f_{1}$ and $f_{2}$. The particular case in which the two feed-centers coincide leads to the case shown in Fig. 1b, where the surface wave is launched by the same feed at two different frequencies. Conversely, the second approach (Fig. 1c) is suited to a single feed-point, after which the signals can be separated using a diplexing circuit. The modulation in Fig. 1c is sequential by gradually switching between different periods: while the internal disk dominantly matches the SW wavelength at the high frequency band, the external annular 
region matches the SW wavelength at low frequency band. However, the period varies as a continuous function from the internal to the external region, to optimize radiation at both sub-bands.

The approach based on overlapping modulations is described first. Although impenetrable impedance boundary conditions (IBCs) may be used, it was shown in [23] that working with the sheet IBC allows one to exert a higher degree of control on the SW-to-LW transformation (see inset of Fig. 1, where sub-wavelength patches are homogenized using a continuous impedance tensor). Provided that the cladding is placed at the air-dielectric interface of an infinite grounded dielectric slab, the boundary sheet imposes at the $\mathrm{z}=0$ plane the condition $\mathbf{E} t=j \underline{\mathbf{X}} \cdot \mathbf{J}$, where $\mathbf{J}=\hat{\mathbf{z}} \times\left(\left.\mathbf{H}_{t}\right|_{z=0^{+}}-\left.\mathbf{H}_{t}\right|_{z=0^{-}}\right)$is the current flowing in the sheet impedance $j \underline{\underline{\mathbf{X}}}$ and $\mathbf{E}_{t}$ is the continuous field at the MTS. The tensor $\underline{\underline{\mathbf{X}}}$ (Hermitian in lossless surfaces and symmetric for highly symmetrical textures) represents the sheet transition reactance. The analyzed modulated MTSs consist of circular domains of radius $a$. Thus, it is convenient to describe the MTS quantities in cylindrical coordinates $(\rho, \varphi)$, with unit vectors $(\hat{\boldsymbol{\rho}}, \hat{\boldsymbol{\varphi}})$. Accordingly, the observation vector is $\boldsymbol{\rho}=\rho \cos \varphi \hat{\mathbf{x}}+\rho \sin \varphi \hat{\mathbf{y}}$. The objective aperture field at frequency $f_{n}$ can be written as $\mathbf{E}^{n}(\boldsymbol{\rho})=\mathbf{e}_{n} E_{0} e^{-\mathrm{j} k_{n} \hat{\mathbf{r}}_{n} \cdot\left(\boldsymbol{\rho}-\boldsymbol{\rho}_{n}\right)} e(\rho) U_{A}(\rho) \quad n=1,2$, where $e(\rho)$ represents the amplitude taper and $U_{A}(\rho)=u(a-\rho)$ is unity when $\rho \leq a$ and zero elsewhere (being $u(x)$ the unit-step function). In turn, $k_{n}$ is the free-space wavenumber at $f_{n}$, and $\hat{\mathbf{r}}_{n}$ and $\mathbf{e}_{n}$ describe the pointing direction and the polarization of beam \#n, respectively. For right-handed circularly polarized (RHCP) broadside pencil beams, we have $\hat{\mathbf{r}}_{1}=\hat{\mathbf{r}}_{2}=\hat{\mathbf{z}}$ and $\mathbf{e}_{1}=\mathbf{e}_{2}=\hat{\mathbf{x}}+j \hat{\mathbf{y}}$. Since a different point source may be defined for each beam, it is also convenient to define local reference systems centered at each point source (see Fig. 1a). Hence, the observation point with respect to the feed positions $\boldsymbol{\rho}_{n}$ is defined as $\mathbf{R}_{n}=\boldsymbol{\rho}-\boldsymbol{\rho}_{n}$, with $n=1,2$. We also define the radial and azimuthal unit vectors $\hat{\boldsymbol{\kappa}}_{n}=\left(\boldsymbol{\rho}-\boldsymbol{\rho}_{n}\right) / R_{n}$ and $\hat{\gamma}_{n}=\left(\hat{\mathbf{z}} \times \hat{\boldsymbol{\kappa}}_{n}\right)$. One can prove (see [7] and the supplementary material) that the entries of $\underline{\underline{\mathbf{X}}}^{n}$ that provide the field distribution $\mathbf{E}^{n}$ at the frequency $f_{n}$, referred to the local coordinate system that originates in $\boldsymbol{\rho}_{n}$, (inset of Fig. 1a) reads as

$$
\begin{aligned}
& X_{\kappa \kappa}^{(n)}\left(\mathbf{R}_{n}\right)=X_{n}\left(f_{n}\right)\left[1+m_{\kappa}^{(n)}\left(\mathbf{R}_{n}\right) \sin \left(\beta_{s w, n} \kappa_{n}-k_{n} \hat{\mathbf{r}}_{n} \cdot \boldsymbol{\kappa}_{n} \pm \Phi_{\kappa}^{(n)}\right)\right] \\
& X_{\kappa \gamma}^{(n)}\left(\mathbf{R}_{n}\right)=X_{n}\left(f_{n}\right) m_{\gamma}^{(n)}\left(\mathbf{R}_{n}\right) \sin \left(\beta_{s w, n} \kappa_{n}-k_{n} \hat{\mathbf{r}}_{n} \cdot \boldsymbol{\kappa}_{n} \pm \Phi_{\gamma}^{(n)}\right) \\
& X_{\gamma \gamma}\left(\mathbf{R}_{n}\right)=\left[X_{n}\left(f_{n}\right) /\left|\hat{\mathbf{e}}_{n} \cdot \hat{\mathbf{\kappa}}_{n}\right|^{2}\right]\left[1-m_{\kappa}^{(n)}\left(\mathbf{R}_{n}\right) \sin \left(\beta_{s w, n} \kappa_{n}-k_{n} \hat{\mathbf{r}}_{n} \cdot \boldsymbol{\kappa}_{n} \pm \Phi_{\kappa}^{(n)}\right)\right]
\end{aligned}
$$

where $m_{\chi}^{(n)}\left(\mathbf{R}_{n}\right)=m\left(\mathbf{R}_{n}\right)\left|\hat{\mathbf{e}}_{n} \cdot \hat{\chi}_{n}\right|$ and $\Phi_{\chi}^{(n)}=\tan ^{-1}\left[\operatorname{Im}\left(\hat{\mathbf{e}}_{n} \cdot \hat{\chi}_{n}\right) / \operatorname{Re}\left(\hat{\mathbf{e}}_{n} \cdot \hat{\chi}_{n}\right)\right]$, with $\chi=\kappa, \gamma$. Furthermore, $X_{n}\left(f_{n}\right)$ and $\beta_{s w, n}$ in (1) denote the average transparent impedance and the SW wavenumber at the frequency $f_{\mathrm{n}}$, respectively. The scalar quantity $m_{\kappa}^{(n)}\left(\mathbf{R}_{n}\right)$ is the modulation index function.

Equation (1) provides the individual modulation $\underline{\underline{\mathbf{X}}}^{n}$ required to radiate beam \#n at $f_{n}$. Modulation \#1 will produce at $f_{2}$ a radiated field quasi-orthogonal (ideally null) to the one produced by modulation \#2 at $f_{2}$, and vice-versa. Bearing this in mind, one can carry out, by virtue of the quasi-orthogonality of such beams, the superposition of both modulations as $\underline{\underline{\mathbf{X}}}=\frac{1}{2} \sum_{n=1}^{2}\left(\hat{\mathbf{\kappa}}_{n} \hat{\mathbf{\kappa}}_{n} X_{\kappa \kappa}^{(n)}+\left(\hat{\boldsymbol{\kappa}}_{n} \hat{\gamma}_{n}+\hat{\gamma}_{n} \hat{\boldsymbol{\kappa}}_{n}\right) X_{\kappa \gamma}^{(n)}+\hat{\boldsymbol{\varphi}}_{n} \hat{\boldsymbol{\varphi}}_{n} X_{\gamma \gamma}^{(n)}\right) U_{A}$. This summation is illustrated in Fig. 1a, where the two added modulations yield an interference that is discriminated afterwards by the individual excitation. When the origin is the 
same for both modulations, the excitation frequency is used for discrimination. The latter case can be studied as a $2 \mathrm{D}$ rectilinear-modulation excited by a planar SW wavefront that locally matches the double periodicity [11]. This canonical problem allows one to estimate the leakage constant $\alpha$ of the -1 mode from the modulation parameters. The indexes $m_{\chi}^{(n)}\left(\mathbf{R}_{n}\right)$ are determined in the design phase from the profile of $\alpha$ that provides a uniform power density distribution at the synthesis frequency $\left(f_{1}+f_{2}\right) / 2$.

In the sequential modulation approach, the aperture is divided in two parts by a circumference of radius $a_{0}$. The period $d(\rho)$ of the modulation increases along the radial distance to match the SW wavelength at two different frequencies. The periodicity function should be such that the region $\rho \in\left(0, a_{0}\right)$ radiates a RHCP broadside beam at the higher frequency $f_{2}$ and the region $\rho \in\left(a_{0}, a\right)$ radiates a RHCP broadside beam at the lower frequency $f_{1}$ (see Fig. 1c). Although the most natural choice is to use different periods in each region, this is not the optimal solution. It is indeed better to define the period as a continuous function of the radius and optimize this function to get the maximum gain in the two sub-bands. The optimized reactance tensor (see [8]) in this sequential approach reads as:

$$
\underline{\underline{\mathbf{X}}}=\bar{X}_{\rho}\left\{\underline{\underline{\mathbf{I}}}+\left[\cos [\Phi(\rho)+\varphi]\left(m_{\rho}(\rho) \hat{\rho} \hat{\rho}-m_{\varphi}(\rho) \hat{\varphi} \hat{\varphi}\right)-m_{\rho}(\rho) \sin [\Phi(\rho)+\varphi](\hat{\rho} \hat{\varphi}+\hat{\varphi} \hat{\rho})\right]\right\}
$$

where the phase of the modulation in (2) is defined as $\Phi(\rho)=\int_{0}^{\rho}\left[2 \pi / d\left(\rho^{\prime}\right)\right] d \rho^{\prime}+\Phi(0)$, with $d(\rho)=\Phi^{\prime}(\rho)$ representing the $\rho$-dependent local period (see [13]). $\bar{X}_{\rho}$ is the average reactance, and $m_{\rho}, m_{\varphi}$ are the radiallydependent modulation indexes. We note that by adopting a non-uniform periodicity function $d(\rho)$ along the radial distance, the reactance $\underline{\underline{\mathbf{X}}}$ in (2) assumes a spiral shape that unwinds outward with a local expansion rate that is dictated by $\Phi(\rho)$. The design strategy optimizes both $d(\rho)$ and the modulation indexes to maximize the gain in the two desired sub-bands. The process starts by defining a set of control points $\rho_{n}^{(1)} \in\left(a_{0}, a\right)$ and $\rho_{n}^{(2)} \in(0, a)$ with $n=1 \ldots N$, the objective sub-bands $\left(\begin{array}{cc}(1) & (1) \\ \min \end{array}, \begin{array}{l}\max \end{array}\right)$ and $\left(\begin{array}{cc}(2) & (2) \\ \min \end{array}, \begin{array}{l}\max \end{array}\right)$ and the desired average gains $G^{(1)}$ and $G^{(2)}$ in each band. The control points $\rho_{n}^{(m)}$ are radii monotonically increasing with $n$ in each subregion. Next, one selects $N$ equally spaced angular frequencies $\omega_{n}^{(m)}$ within each sub-band, and connects each $\omega_{n}^{(m)}$ with the SW wavelengths values $\lambda_{s w, n}^{(m)}=2 \pi / \beta_{s w, n}^{(m)}$ obtained from the dispersion curve of the SW zero-indexed mode over the average uniform reactance $\bar{X}_{\rho}$. Each set $\rho_{n}^{(m)}$ is associated to $\lambda_{s w, n}^{(m)}$ through $d\left(\rho_{n}^{(m)}\right)=\lambda_{s w, n}^{(m)}$. Thus, by changing $\rho_{n}^{(m)}$ one can optimize the continuous periodicity function $d(\rho)$ and, therefore, the phase of the aperture field. Furthermore, a constant power density distribution of the -1 mode, has to be imposed at the synthesis angular frequency $\omega_{\text {amp }}$, which is also adjusted at each optimization step. The radius $a_{0}$ is also changed at each step, and not fixed a priori, to obtain an additional degree of freedom. The cost function for the optimization algorithm is defined as the average distance between the calculated gain $G\left(\omega_{n}^{(m)}\right)$ and the expected constant gain $G^{(m)}$ within each sub-band, namely

$$
C=\sum_{n, m} \frac{\left|G\left(\omega_{n}^{(m)}\right)-G^{(m)}\right|}{G^{(m)}} .
$$


At each iteration of the algorithm, the position of the control points $\rho_{n}$ are adaptively modified along the radius by a swarm optimization search to minimize the cost function. The positions of the control points $\rho_{n}$ do not change their increasing order with $n$ to ensure that $d(\rho)$ is monotonically increasing with $\rho$ at any iteration. To calculate the cost function according with (3), it is necessary to compute the gain as a function of frequency at each optimization step. To this end, we use the flat-optics [23] formula

$$
G(\omega)=\frac{8 \pi^{2}}{\lambda^{2}} \frac{\left|\int_{0}^{a} \sqrt{S(\rho, \omega)} e^{j \Psi(\rho, \omega)} \rho d \rho\right|^{2}}{\int_{0}^{a} S(\rho, \omega) \rho d \rho}
$$

where $S(\rho, \omega)$ in (4) is the power density function per unit surface and $\Psi(\rho, \omega)=\Phi(\rho)-\beta_{s w} \rho-\int_{0}^{\rho} \Delta \beta_{s w}\left(\rho^{\prime}\right) d \rho^{\prime}$ is the phase of the -1 indexed modal field component of the adiabatic Floquet wave expansion [23]. Furthermore, $\Delta \beta_{s w}$ accounts for an incremental deviation of the SW wavenumber $\beta_{s w}$ due to the impedance modulation around its average $\bar{X}_{\rho}$. The latter is determined as in [8] by solving the periodic canonical problem that locally matches the local period. At frequency $\omega_{a m p}, S\left(\rho, \omega_{\text {amp }}\right)$ is a uniform function with a rapid drop-off at the rim of the aperture. As explained in the Appendix of [8], one can retrieve the local attenuation constant $\alpha\left(\rho, \omega_{\text {amp }}\right)$ of the 1 mode from $S\left(\rho, \omega_{\text {amp }}\right)$ using

$$
\alpha\left(\rho, \omega_{\text {amp }}\right)=\frac{\rho S\left(\rho, \omega_{\text {amp }}\right) / 2}{\frac{1}{2 \pi} P_{s w}-\int_{o}^{\rho} S\left(\rho^{\prime}, \omega_{\text {amp }}\right) \rho^{\prime} d \rho^{\prime}}
$$

where $P_{s w}$ is the power associated to the SW. Such information on $\alpha\left(\rho, \omega_{a m p}\right)$, combined with the solution of the local canonical problem [23], allows one to determine the relevant modulation indexes $m_{\rho}, m_{\varphi}$ that best match the $\alpha\left(\rho, \omega_{\text {amp }}\right)$ profile obtained by (5). The estimate of $\alpha(\rho, \omega)$ at any frequency of the two sub-bands is obtained owing to a priory knowledge of the function $j \bar{X}_{\rho}(\omega)$, which is obtained from the reactance of the patches used to synthesize (2) in the frequency range of interest. Once $\alpha(\rho, \omega)$ is obtained, we can compute the function $S(\rho, \omega)$ at any frequency with the inversion formula of (5) using (26) in [8]

$$
S(\rho, \omega)=2 \alpha(\rho, \omega) \frac{1}{\rho} \frac{P_{S W}}{2 \pi} \exp \left(-2 \int_{0}^{\rho} \alpha\left(\rho^{\prime}, \omega\right) d \rho^{\prime}\right)
$$

Using (6) in (4), one may calculate the gain at each frequency of the two sub-bands.

The remainder of this letter presents some selected numerical and experimental results. Fig. 2 presents the performance of two single-feed antennas with $a=10 \mathrm{~cm}$ designed to work around 26 and $32 \mathrm{GHz}$ by the overlapped (Fig.1b) and sequential modulation methods (Fig 1c). The objective gains in the optimization of the sequential modulation were $30 \mathrm{~dB}$ at $26.25 \mathrm{GHz}$ and $32 \mathrm{~dB}$ at $32.05 \mathrm{GHz}$. An aperture efficiency upper bound has not been investigated. However, we have empirically verified that sequential modulations typically provide aperture efficiencies below $30 \%$, while the overlapped approach may yield efficiencies $>35 \%$. The aperture efficiency is impaired in both cases by the MTS dispersion. The uniform power density function imposed on the aperture at the synthesis frequency $\left(\omega_{\text {amp }} / 2 \pi\right.$ for sequential modulations and $\left(f_{1}+f_{2}\right) / 2$ for overlapped ones) undergoes a variation with frequency: it diverges from the ideal uniform function as we move away from the synthesis frequency, leading to a reduction of the aperture efficiency. Moreover, although the dominating effect at $f_{1}$ in the overlapped (sequential) approach is the broadside beam generated by modulation \#1 (active region \#1), modulation \#2 (active region \#2) will also contribute (weakly) to the 
total radiation at $f_{1}$, generating undesired side-lobes, and the other way around at $f_{2}$. These sidelobes further reduce the aperture efficiency. Overlapped modulations designs partially balance these effects by exploiting the whole aperture.

The impedance tensors have been implemented at the synthesis frequency by elliptical sub-wavelength patches [24] printed on a grounded substrate with $\varepsilon_{\mathrm{r}}=6.15$ and $h=640 \mu \mathrm{m}$. The fundamental design parameters are reported in the caption of Fig.2. The full-wave analysis of the resulting structures is based on a Fast Multipole Method (FMM) [11] and on the Method of Moments (MoM) solution of the continuous impedance boundary condition (IBC) problem [25]. In this second model, the mathematical impedance described in (1) and (2) is applied in the definition of the integral equation; an elementary dipole at the interface is used for excitation. Concerning the FMM results, about 17000 elliptical patches have been used for synthesizing the impedance for both types of modulation (see inset in Fig. 2). The presented results include the directivity patterns as a function of the elevation angle and the maximum directivity as a function of frequency in the two sub-bands. Fig 2 also reports the periodicity functions obtained in each case. The best performance is obtained for the overlapped modulation case, especially for lower frequencies. Indeed, the sequential approach at $f_{1}=26 \mathrm{GHz}$ presents higher sidelobes due to both the spurious radiation of the non-active circular region and the annular shape of the active region (the Hankel transform of an annulus with uniform amplitude presents higher sidelobe levels and a narrower beam than from the equivalent circular area) [13]-[14].

Fig. 3 presents measurements for a $10 \mathrm{~cm}$ radius antenna with overlapped modulations, working at $25 \mathrm{and} 30 \mathrm{GHz}$ and two feed points separated by a distance of $4.1 \mathrm{~cm}$. The figure shows the normalized radiation patterns at these two frequencies on the plane orthogonal to the alignment of the two feeds, and the scattering parameters. The radiation patterns reasonably meet the expectations. The input parameters present a good matching for both ports at the design frequencies, and the average isolation level between ports is around $27 \mathrm{~dB}$ over a wide band.

This letter has presented two design methods for bichromatic MTS antennas at Ka-band. By controlling the power density distribution on the aperture, and owing to the weak interaction between the SW excitation and modulation (active region) \#2 at $f_{1}$ and modulation (active region) \#1 at $f_{2}$, the overlapped (sequential) modulation method can provide aperture efficiencies $>35 \%(\approx 30 \%)$. Both methods yield a good polarization control and moderate sidelobes. Full-wave simulations and measurements validate the proposed approaches. 

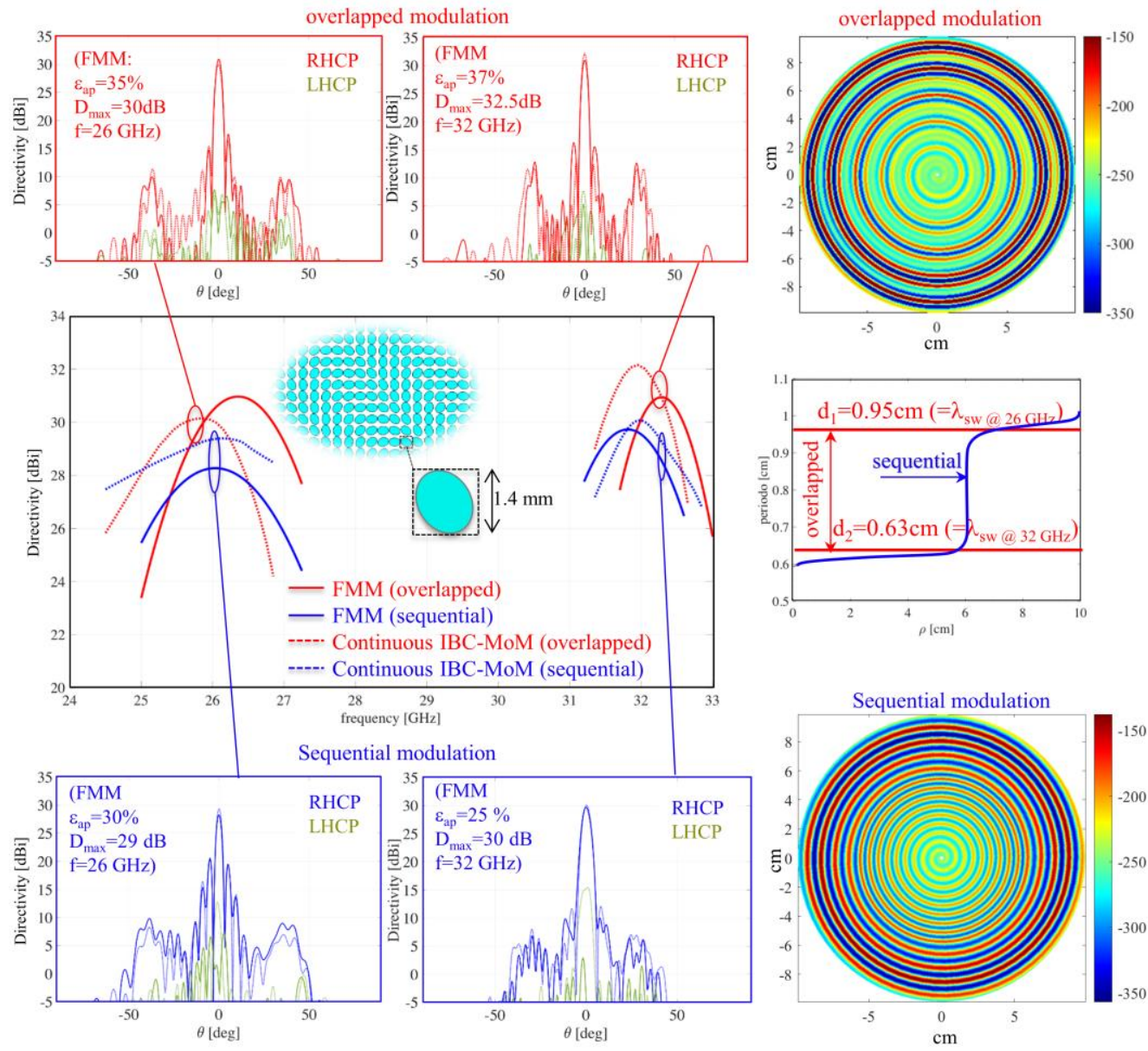

Fig. 2 Full-wave numerical results obtained by Fast Multiple Method (FMM, continuous lines) and Continuous IBC Method of Moments (dashed lines). The radiation pattern for both overlapped modulation (red lines, on top) and sequential modulation (blue lines, on bottom) have been calculated for an antenna of $10 \mathrm{~cm}$ radius optimized for a gain of about $30 \mathrm{~dB}$ at $26.25 \mathrm{GHz}$ and $32 \mathrm{~dB}$ at 32.05GHz. The FMM results are obtained for a layout constituted by small elliptical patches located in a square lattice of periodicity $1.4 \mathrm{~mm}$, and printed on a $640 \mu \mathrm{m}$ thick grounded substrate with relative permittivity 6.15 . At the center of the figure: maximum directivity as a function of frequency. On the right-hand side: colored plots of the modulation amplitude of the radial component of the impedance tensor for the two cases (top: sequential modulation; bottom: overlapped modulation). For the sequential modulation the period is gradually varied along the radius, and it exhibits a rapid change around $a_{0}=6 \mathrm{~cm}$. The period along the radius is also presented. The overlapped case uses two constant periods chosen to match the SW-wavelength obtained at $f_{1}$ and $f_{2}$. 

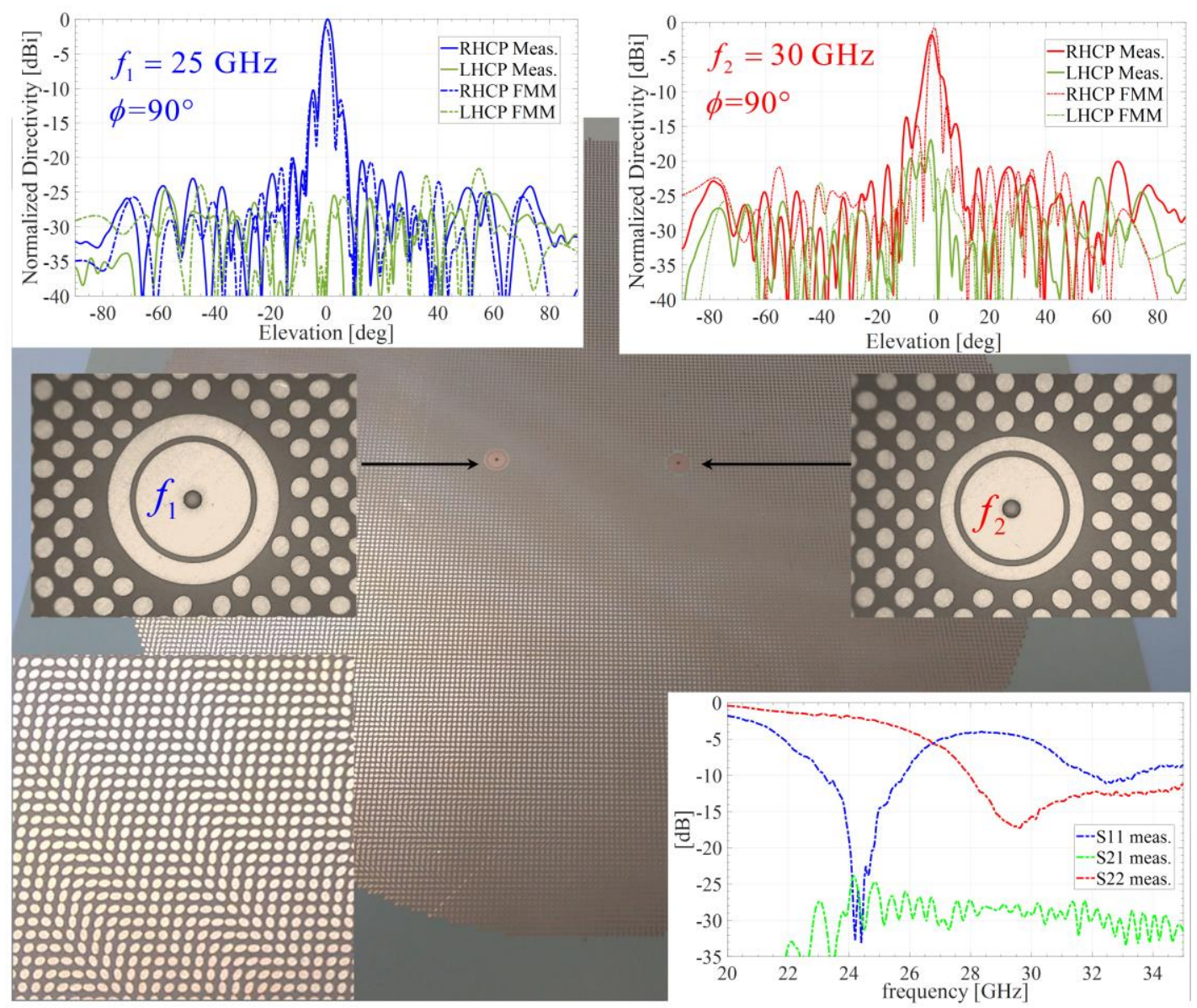

Fig. 3 Prototype of a $a=10 \mathrm{~cm}$ radius MTS antenna for bi-chromatic beam generation using two different feed points. The MTS is constituted by small elliptical patches embedded in a square lattice of $1.0 \mathrm{~mm}$ periodicity, and printed on a grounded substrate of thickness $640 \mu \mathrm{m}$ and relative permittivity 10.2. The two feeders are separated by a distance of $4.1 \mathrm{~cm}$, they are constituted by a vertical monopole with a circular slot-loaded patch (printed on the same MTS substrate) on top. In the insets: details of the two feeders and measured (continuous line) and simulated (dashed lines) radiation patterns at $f_{1}=25 \mathrm{GHz}$ and $f_{2}=30 \mathrm{GHz}$ on the plane orthogonal to the alignment of the two feeds. In the bottom-right corner: measured two-port scattering parameters. The full-wave simulations yield total efficiencies (including Ohmic and dielectric losses) of $-1.8 \mathrm{~dB}$ and $-2.8 \mathrm{~dB}$ at $25 \mathrm{GH}$ and $30 \mathrm{GHz}$, respectively.

\section{SUPPLEMENTARY MATERIAL}

This section describes how the entries of the impedance tensor in (1) can be obtained from the objective aperture field $\mathbf{E}^{n}(\boldsymbol{\rho})$.

The authors acknowledge funding from "Conseil Régional de Bretagne" through the grant SAD17012, and from "Ministero dell'Istruzione, dell'Università e della Ricerca (MIUR)" through the PRIN project 2017S29ZLA.

\section{DATA AVAILABILITY}

The data that support the findings of this study are available from the corresponding author upon reasonable request.

\section{REFERENCES}

[1] C. Holloway, E. F. Kuester, J. Gordon, J. O'Hara, J. Booth, and D. Smith, "An overview of the theory and applications of metasurfaces: The two-dimensional equivalents of metamaterials," IEEE Antennas Propag. Mag. 54, 10-35 (2012).

[2] A. Patel and A. Grbic, "Transformation electromagnetics devices based on printed-circuit tensor impedance surfaces," IEEE Trans. Microw. Theory Tech. 62, 1102-1111 (2014).

[3] M. Mencagli, E. Martini, D. González-Ovejero, and S. Maci, "Metasurfing by transformation electromagnetics," IEEE Antennas Wireless Propag. Lett. 13, 1767-1770 (2014).

[4] C. Pfeiffer and A. Grbic, "Metamaterial huygens' surfaces: Tailoring wave fronts with reflectionless sheets," Phys. Rev. Lett. 110, 197401 (2013).

[5] V. S. Asadchy, M. Albooyeh, S. N. Tcvetkova, A. Díaz-Rubio, Y. Ra'di, and S. A. Tretyakov, "Perfect control of reflection and refraction using spatially dispersive metasurfaces," Phys. Rev. B 94, 075142 (2016). 
[6] B. Fong, J. Colburn, J. Ottusch, J. Visher, and D. Sievenpiper, "Scalar and tensor holographic artificial impedance surfaces," IEEE Trans. Antennas Propag. 58, 3212-3221 (2010).

[7] G. Minatti, M. Faenzi, E. Martini, F. Caminita, P. De Vita, D. González-Ovejero, M. Sabbadini, and S. Maci, "Modulated metasurface antennas for space: Synthesis, analysis and realizations," IEEE Trans. Antennas Propag. 63, 1288-1300 (2015).

[8] G. Minatti, F. Caminita, E. Martini, M. Sabbadini, and S. Maci, "Synthesis of modulated-metasurface antennas with amplitude, phase and polarization control,” IEEE Trans. Antennas Propag. 64, 3907-3919 (2016).

[9] D. González-Ovejero, N. Chahat, R. Sauleau, G. Chattopadhyay, S. Maci and M. Ettorre, "Additive manufactured metal-only modulated metasurface antennas," IEEE Trans. Antennas Propag. 66, 6106-6114 (2018).

[10] G. Minatti, E. Martini, and S. Maci, “Efficiency of metasurface antennas,” IEEE Trans. Antennas Propag. 65, 1532-1541 (2017).

[11] M. Faenzi, G. Minatti, D. González-Ovejero, F. Caminita, E. Martini, C. Della Giovampaola, and S. Maci, "Metasurface antennas: new models, applications and realizations," Sci. Rep. 9, 1-14 (2019).

[12] G. Minatti, M. Faenzi, M. Sabbadini and S. Maci, "Bandwidth of gain in metasurface antennas,” IEEE Trans. Antennas Propag. 65, 2836-2842 (2017).

[13] M. Faenzi, D. González-Ovejero, and S. Maci, "Wideband active region metasurface antennas," IEEE Trans. Antennas Propag. 68, $1261-1272$. (2019).

[14] M. Faenzi, D. González-Ovejero and S. Maci, "Flat gain broad-band metasurface antennas," IEEE Trans. Antennas Propag., in press.

[15] A. Tellechea, E. Martini, D. Gonzalez-Ovejero, M. Faenzi, G. Minatti and S. Maci, "Dual band isoflux ultraflat meta antennas," Proc. 9th Eur. Conf. Antennas Propag., 1-5 (2015).

[16] D. González-Ovejero, G. Minatti, G. Chattopadhyay and S. Maci, "Multibeam by metasurface antennas," IEEE Trans. Antennas Propag. 65, 2923-2930 (2017).

[17] M. Faenzi, D. González-Ovejero, F. Caminita, and S. Maci, "Dual-band self-diplexed modulated metasurface antennas". 12th Eur. Conf. Antennas Propag., 1-4 (2018)

[18] Y. Li, A. Li, T. Cui, and D. F. Sievenpiper, "Multiwavelength multiplexing hologram designed using impedance metasurfaces," IEEE Trans. Antennas Propag. 66, 6408-6413. (2018).

[19] M. Bodehou, C. Craeye and I. Huynen, "Multifrequency band synthesis of modulated metasurface antennas," IEEE Antennas Wireless Propag. Lett. 19, 134-138 (2020).

[20] M. Bodehou, C. Craeye, E. Martini and I. Huynen, "A quasi-direct method for the surface impedance design of modulated metasurface antennas," IEEE Trans. Antennas Propag. 67, 24-36 (2019).

[21] M. Bodehou, E. Martini, S. Maci, I. Huynen and C. Craeye, "Multibeam and beam scanning with modulated metasurfaces," IEEE Trans. Antennas Propag. 68, 1273-1281 (2020).

[22] J. Deng, Y. Yang, J. Tao, L. Deng, D. Liu, Z. Guan, G. Li, Z. Li, S. Yu, G. Zheng, Z. Li, and S. Zhang, "Spatial frequency multiplexed metaholography and meta-nanoprinting," ACS Nano 13, 9237-9246 (2019).

[23] G. Minatti, F. Caminita, E. Martini, and S. Maci, "Flat optics for leaky-waves on modulated metasurfaces: adiabatic Floquet-wave analysis," IEEE Trans. Antennas Propag. 64, 3896-3906 (2016).

[24] M. Mencagli Jr., E. Martini, and S. Maci, "Surface wave dispersion for anisotropic metasurfaces constituted by elliptical patches," IEEE Trans. Antennas Propag. 63, 2992-3003 (2015).

[25] D. González-Ovejero and S. Maci, "Gaussian ring basis functions for the analysis of modulated metasurface antennas," IEEE Trans. Antennas Propag. 63, 3982-3993 (2015). 

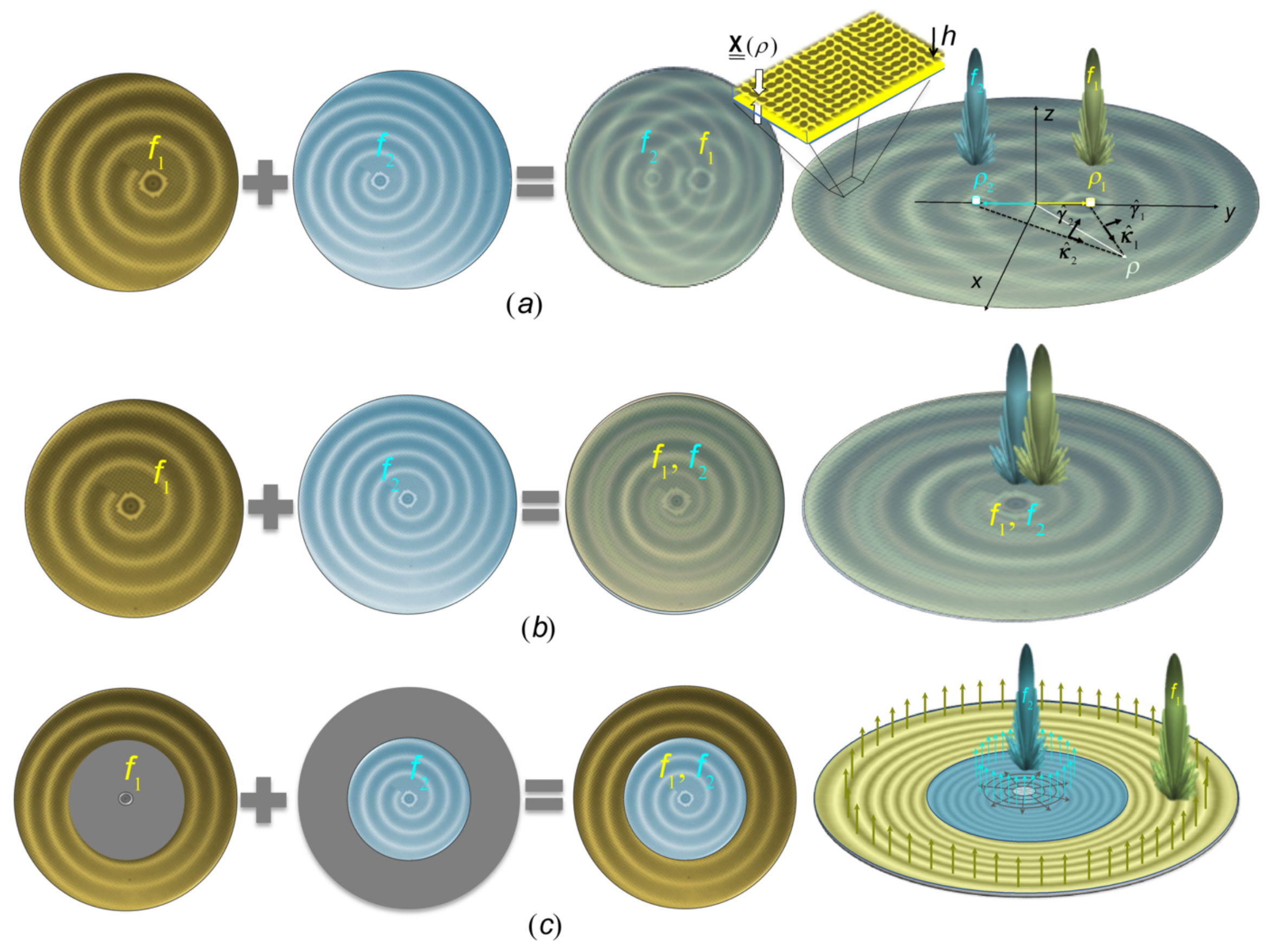
overlapped modulation
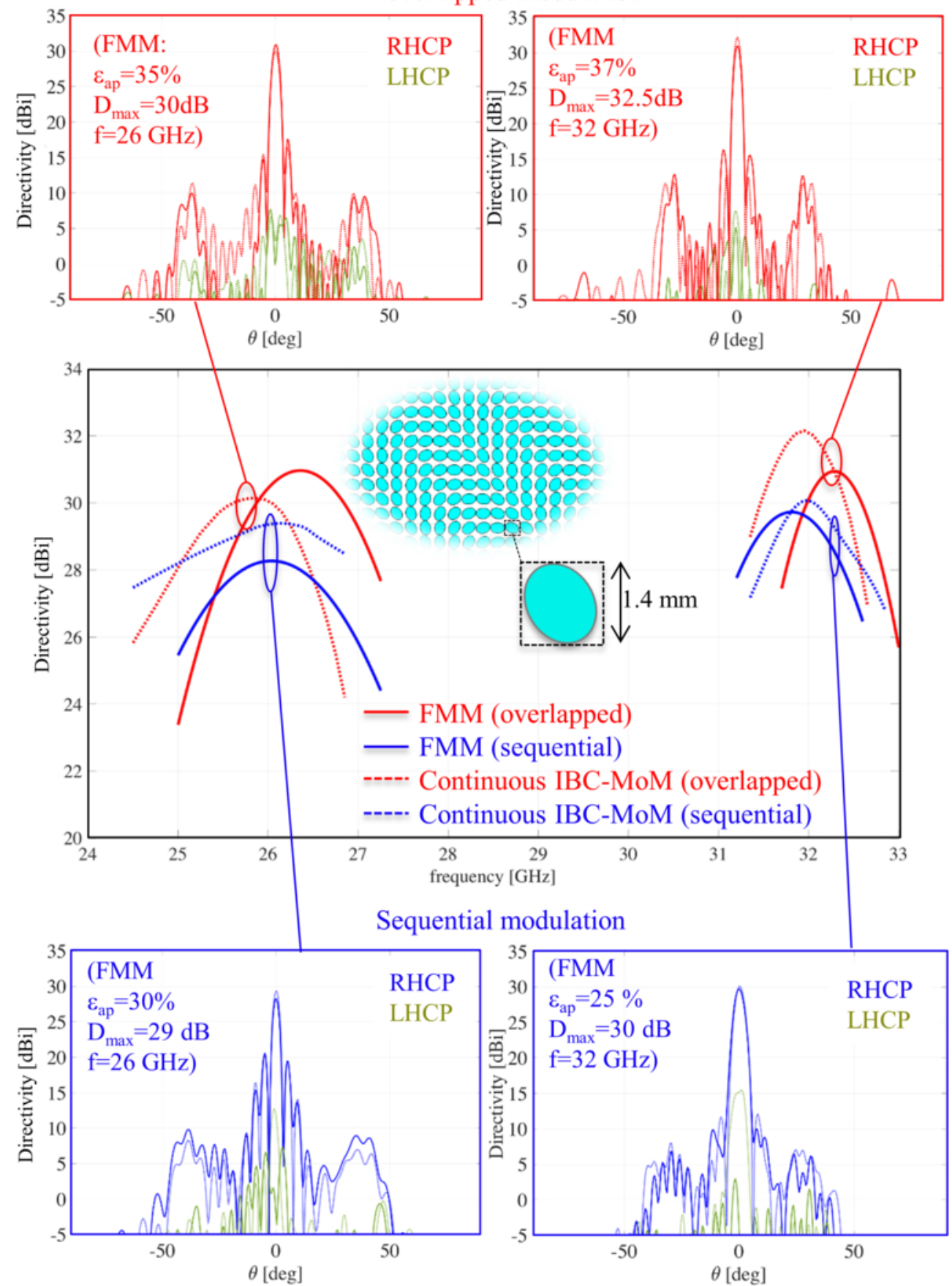
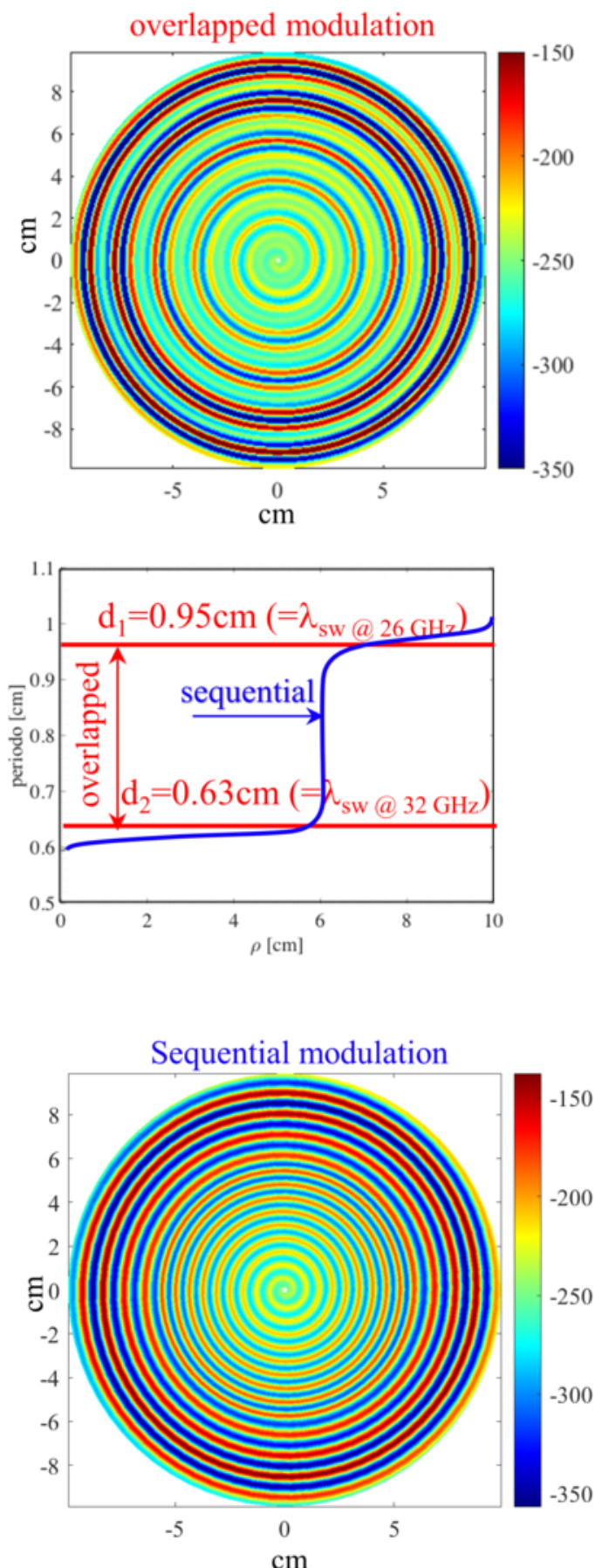

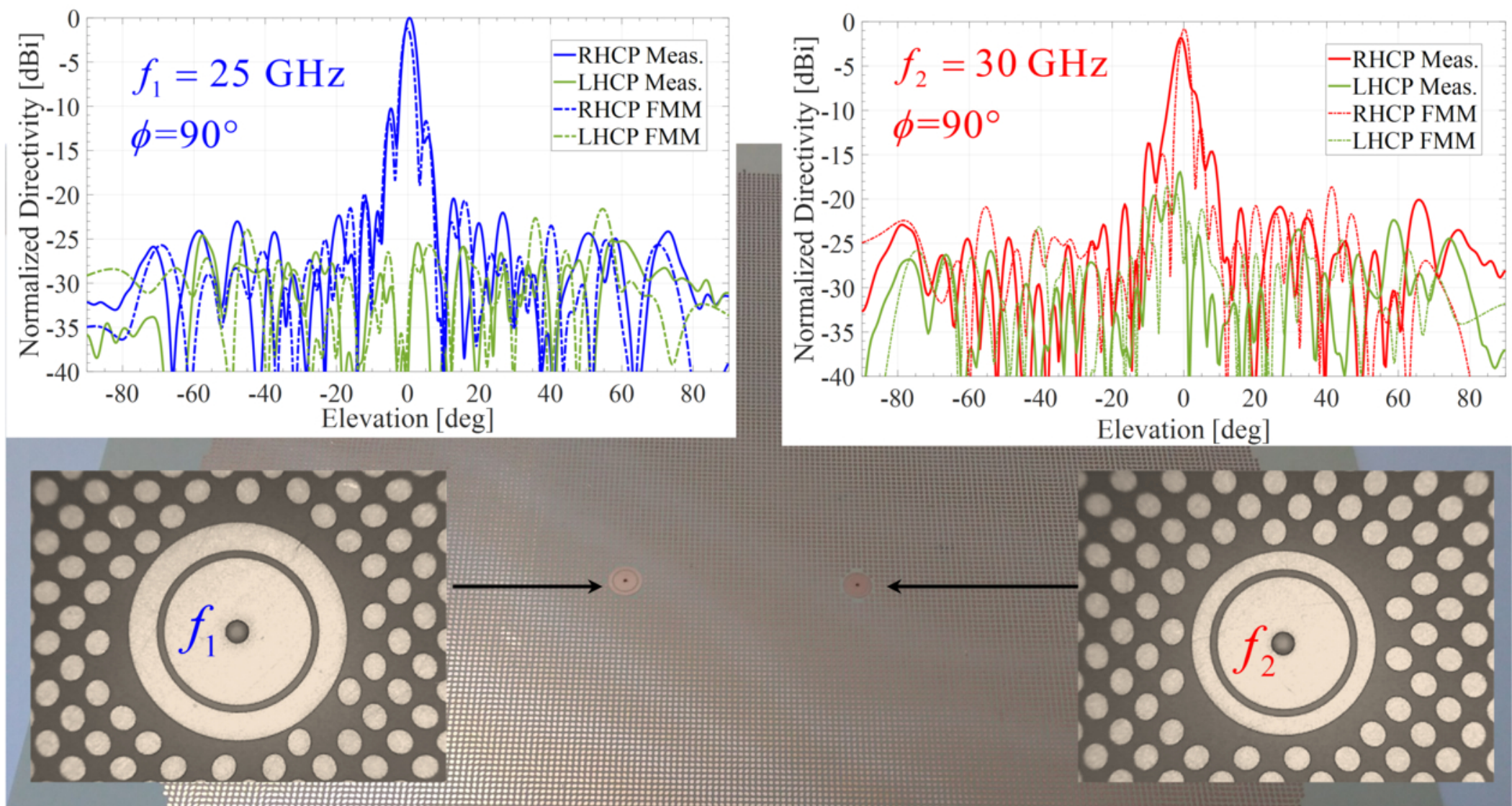

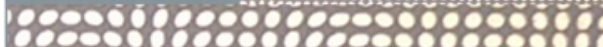
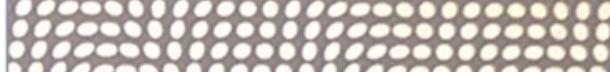

  

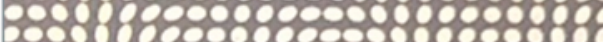

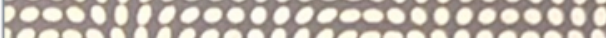

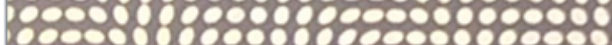

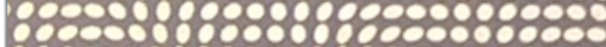

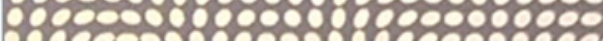
   00000000000001000001

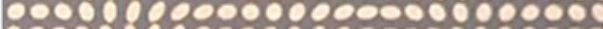
00000 1 2000006 6000600000000  

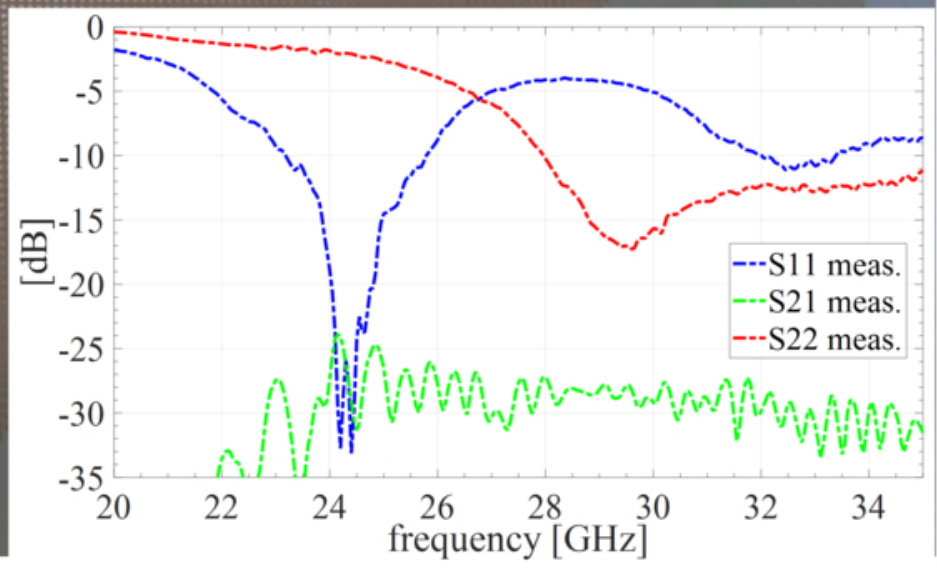

